\title{
Editorial
}

\section{Use of artificial pancreas in management of diabetes mellitus}

\author{
Amit Kumar Sharma ${ }^{1}$, Sheikh Yasir Islam ${ }^{1}$, , Lalit Gupta ${ }^{\circledR 2}$ \\ ${ }^{1}$ Dept. of Medicine, Lady Hardinge Medical College and S S K Hospital, New Delhi, India \\ ${ }^{2}$ Dept. of Anaesthesia, Maulana Azad Medical College and Associated Hospital, New Delhi, India
}

\section{A R T I C L E I N F O}

\section{Article history:}

Received 18-01-2022

Accepted 19-01-2022

Available online 12-02-2022

\begin{abstract}
This is an Open Access (OA) journal, and articles are distributed under the terms of the Creative Commons Attribution-NonCommercial-ShareAlike 4.0 License, which allows others to remix, tweak, and build upon the work non-commercially, as long as appropriate credit is given and the new creations are licensed under the identical terms.

For reprints contact: reprint@ipinnovative.com
\end{abstract}

\section{Introduction}

Insulin injection has been the main treatment in patients with Type 1 Diabetes Mellitus and many Type 2 DM patients. However, despite concerted efforts of patients and physicians/caregivers, control of blood glucose has often been difficult to achieve Artificial pancreas (AP) is a closed-loop system combining a glucose sensor, a control algorithm, and an insulin infusion device. AP developments can be traced back 50 years to when the possibility for external blood glucose regulation was established by studies in individuals with type 1 diabetes using intravenous glucose measurement and infusion of insulin and glucose. ${ }^{1}$

Physicians, scientists, and engineers have worked enormously in past few decades to develop complex medical devices for patients with diabetes that emulate the pancreatic regulation of glucose in otherwise healthy individuals. Research groups in Canada, Germany, France, Australia, and Japan in the 1970s and 1980s were able to demonstrate proof of principle of the feasibility of automated control of glucose. ${ }^{2,3}$ Albisser et al. were among the first to describe an "artificial endocrine pancreas" in a paper published in $1974 .{ }^{4}$ They noted that the healthy pancreas secretes insulin as required to maintain blood glucose within normal physiologic limits. They referred to an artificial pancreas as a "computerized control system which

\footnotetext{
* Corresponding author.

E-mail address: dramitsharma1506@gmail.com (S. Y. Islam).
}

closely simulates this particular endocrine function of the pancreas." The patient's instantaneous blood glucose was determined from a custom laboratory instrument measuring venous glucose concentration that output the data to a laboratory computer. Algorithms in the computer, using both the instantaneous glucose and the rate of change, then calculated the appropriate infusion of insulin and dextrose needed to maintain the subject's blood glucose within the normal range. These systems relied upon intravascular administration of glucose and insulin or other components with a high level of complexity and could be used only under carefully supervised inpatient conditions. ${ }^{5}$

Recent advances in insulins, insulin pumps, continuous glucose-monitoring systems, and control algorithms have resulted in a dramatic acceleration of progress in the development of artificial pancreas devices ultimately intended for use in the outpatient setting. In the last 3 years alone, there have been numerous excellent reviews of progress made in the field of artificial pancreas research. There are in principle multiple approaches to achieving an artificial pancreas, including the use of fully implanted insulin-infusion systems and fully implanted continuous glucose sensors. The two major system-level approaches to achieve closed-loop control of blood glucose in diabetic patients are the unihormonal approach using insulin only to lower blood glucose and the bihormonal approach using insulin to lower blood glucose and glucagon to raise blood glucose. The bihormonal approach may be expanded to 
include the use of pramlintide to delay gastric emptying and downregulate endogenous hyperglucagonemia, thereby providing still further improvements in glycemic control. ${ }^{6}$

Strategies for the design of control algorithms and supervision control for application to the artificial pancreas

Proportional-integral-derivative (PID) and fuzzy logic are used for glucose related insulin response. For prevention of hyperglycemia, the hyperglycemia safety system (HSS) module is followed, which uses a combination of the SSM metabolic estimation procedure and an insulin-on-board calculation that compares the actual insulin on board with the ideal insulin on board needed to achieve euglycemia. ${ }^{7}$

Meal safety system (MSS) is used to respond to increases in glucose owing to consumption of a meal. After an announced meal (i.e., after the closed-loop algorithms have been informed by the patient that he or she is eating a meal), if there is no imminent risk of hypoglycemia, the MSS component of the closed-loop algorithm increases the basal rate up to three times the preprogrammed value. Insulin is attenuated by the action of the MSS module after a meal when the glucose decreases (e.g., to $110 \mathrm{mg} / \mathrm{dL}$ ) and the insulin is attenuated. When the continuous glucose monitor detects a rise in glucose, the remaining insulin required for the meal (not yet provided as part of the increased basal infusion of insulin) is infused as a single bolus. ${ }^{8}$

The fourth and final module in the UVA modular system is the hyperglycemia-mitigation system (HMS). The HMS module predicts the estimated glucose 30-60 min forward in time, and if the forecast indicates a glucose value above the hyperglycemia threshold of $180 \mathrm{mg} / \mathrm{dL}$, the HMS uses the insulin-on-board information from the pump and provides a correction bolus calculated as $60 \%$ of the correction bolus needed to achieve euglycemia. HMS can repeat such hyperglycemic correction boluses every hour as needed. ${ }^{9}$

\subsection{Current status in clinical medicine limitations of current sensors and proposed improvements}

Continuous subcutaneous insulin infusion has become an accepted mode of insulin pump therapy. Improvements in safety, miniaturization, refined tuning of insulin pumps allowing for fine adjustments of basal rate, the recently introduced "patch pumps", and new insulin analogs, all lead to improved patient comfort and better glucose control. The key issue of subcutaneous insulin delivery remains the delay in action caused by the time needed for subcutaneous absorption, resulting in late insulin peaks up to $120 \mathrm{~min}$ after the injection of a subcutaneous bolus of regular insulin. Following are the few challenges that needs to be addressed. ${ }^{10}$

1. Most of the commercial subcutaneous CGM devices measure glucose concentration in the interstitium. However, during rapidly changing conditions, e.g., after a meal or during a hypoglycemic episode, interstitial glucose and blood glucose can be markedly different. Thus, CGM devices require calibration using one or more daily blood glucose samples.

2. Time lag exists because of blood-to-interstitial glucose transport and the sensor processing time (instrument delay). Such a time lag could greatly influence the accuracy of CGM and hence for the purpose of closedloop control, mitigation of the time lag was suggested based on near-term glucose forecast methods

3. Errors from transient loss of sensitivity, and random noise confound CGM data. Thus, filtering, denoising, and artifact rejection in CGM data are important for closed-loop control.

Currently available devices in the US are Abbott Navigator, Medtronic Enlite, Dexcom SEVEN PLUS and G4 platinum.

\section{Conclusion}

Development of the artificial pancreas has been intensively pursued over the past few decades. The ultimate goal of an AP system is to improve overall diabetes management and reduce the frequency of life-threatening events associated with diabetes. The algorithms used by the AP to calculate insulin dosage have been intensively investigated, either using data from diabetic patients or computer simulated patients. Artificial intelligence has become more established over the past few years and could ultimately provide better solutions to meet the current limitations of an AP.

\section{Conflicts of Interest}

The authors declare no conflict of interest.

\section{References}

1. Kadish AH. Automation control of blood sugar. I. A servomechanism for glucose monitoring and control. Am J Med Electron. 1964;3:82-6.

2. Clemens AH, Chang PH, Myers RW. The development of Biostator, a glucose controlled insulin infusion system (GCIIS). Horm Metab Res. 1977;(Suppl 7):23-33.

3. Albisser AM. An artificial endocrine pancreas. Diabetes. 1974;23:389-396.

4. Russell SJ. Blood glucose control in type 1 diabetes with a bihormonal bionic endocrine pancreas. Diabetes Care. 2012;35(11):2148-55.

5. Steil GM. Feasibility of automating insulin delivery for the treatment of type 1 diabetes. Diabetes. 2006;55(12):3344-50.

6. Christiansen M. A new-generation continuous glucose monitoring system: improved accuracy and reliability compared with a previousgeneration system. Diabetes Technol Ther. 2013;15(10):881-8.

7. Updike SJ, Shults M, Ekman B. Implanting the glucose enzyme electrode: problems, progress, and alternative solutions. Diabetes Care. 1982;5:207-12.

8. Breton M. Fully integrated artificial pancreas in type 1 diabetes: modular closed-loop glucose control maintains near normoglycemia. Diabetes. 2012;61(9):2230-7.

9. Hughes CS, Patek SD, Breton MD, Kovatchev BP. Hypoglycemia prevention via pump attenuation and red-yellow-green "traffic" lights using continuous glucose monitoring and insulin pump data. $J$ Diabetes Sci Technol. 2010;4(5):1146-55.

10. Aussedat B, Dupire-Angel M, Gifford R, Klein JC, Wilson GS, Reach G. Interstitial glucose concentration and glycemia: implications 
for continuous subcutaneous glucose monitoring. Am J Physiol Endocrinol Metab. 2000;278:716-28.

\section{Author biography}

Amit Kumar Sharma, Associate Professor
Lalit Gupta, Associate Professor (D) https://orcid.org/0000-0001-72915961

Cite this article: Sharma AK, Islam SY, Gupta L. Use of artificial pancreas in management of diabetes mellitus. Indian J Clin Anaesth 2022;9(1):1-3.

Sheikh Yasir Islam, Assistant Professor 